\title{
Spinal cord injury, Africa and women's concerns: sustaining our commitments as we move into the 20 s
}

\author{
Marcalee Alexander ${ }^{1}$
}

Received: 3 January 2020 / Accepted: 10 January 2020

(C) International Spinal Cord Society 2020

\section{Happy New Decade!}

As we move into the future it is always good to be positive about possibilities and things we can do to improve the lives of people living with SCI/D and their friends and families. We are also concerned with those that are most in need. In light of this we are expanding our special foci process from India to Africa and working to highlight reports from Africa this year. We already have some papers that have been submitted and are interested to hear your experiences. In addition, we realize that more information is necessary about the issues of the minority of persons with SCIs, women. So, we are also soliciting case reports, series, and pilot studies pertaining to women's issues. Moreover, for both areas, we would like you to continue to incorporate sustainability in as a focus of your work. Have you considered the reproducibility of what you do? Are you using standard terminology such as the International Standards for the Assessment of Autonomic Function after SCI, the International Data Sets and the International Standards for the Neurologic Classification of SCI? Also, have you considered the future of persons with SCI both locally and internationally in your initiatives and are your proposals relevant and sustainable around the world?

A good example of this is the paper which won our 2019 paper of the year award: Efficacy of water spray for evaporative cooling in athletes with spinal cord injury by Michelle Trbovich, Wouter Koek and Catherine Ortega. This paper focuses on a practical concept and provides information about its utility. The work could easily be transferred into clinical practice and used in nonathletic individuals at a low cost. To me these last two issues are the most important-transferability and low cost. As professionals, we are all committed to our duties and caring for all and water can be used on every continent, so I encourage you all to read this paper and consider the concept as temperatures rise.

In addition to the paper of the year, I would also like to announce the new best poster awards from our meeting in Nice. Thomas Bryce won an award for: The feasibility of performing home evaluations using a mobile application with coauthors Chung-Ying Tsai, Alison Swirsky Miller, Vincent Huang, and Miguel Escalon. Additionally, Ralf Böthig received an award for: Spinal cord injury confers bladder cancer risk: lessons from a comparison of clinical data with the national database with coauthors Kai Fiebag, Birgitt Kowald, Sven Hirschfeld, Roland Thietje, Ines Kurze, Albert Kaufmann, Wolfgang Schöps, Michael Zellner, Holger Böhme, Thura Kadhum, and Klaus Golka.

I am happy to announce additions to the SCSC Editorial Board. Jorge Krebs, Mohit Arora, and Nishu Tyagi will join the editorial board and Dan Graves will join us as an Associate Editor. I would also like to thank Mindy Aisen, Ruth Marshall, John Ditunno, Wagih ElMasry, Lisa Harvey, and Inge Blockmans who are rotating off the board. Finally, remember this is your journal and journals are like a living being. SCSC is here for you and we need your help, any way you would like to participate. Just email me at spinalcordmd@live.com and we will find a way for you to help!
Marcalee Alexander

spinalcordmd@live.com

1 University of Alabama School of Medicine, Birmingham, Birmingham, AL, USA 